\title{
Evaluation of the Clinical and Histological Effectiveness of Isotretinoin in the Therapy of Oral Leukoplakia Ten Years of Experience: Is Management Still Up to Date and Effective?
}

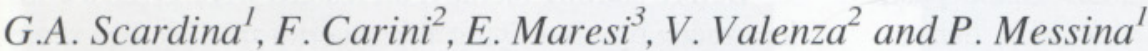

${ }^{1}$ Department of Oral Science, Section of Oral Medicine; ${ }^{2}$ Department of Experimental Medicine, Section of Human Anatomy; ${ }^{3}$ Department of Pathologic Anatomy, University of Palermo, Palermo, Italy

\section{SUMMARY}

Various studies have evaluated the therapentic effectiveness of vitamin A derivatives in the treatment of oral leukoplakia $(O L)$. Not all the studies have shown concordant results. The aim of our study, which has a 10-year follow-up, was to test the effectiveness of topical therapy based on $0.18 \%$ isotretinoin by comparing it with that most frequently used, i.e., at $0.05 \%$ concentration. Forty patients with an established diagnosis of $O L$ were involved in the study. The patients were randomly divided into two groups and the drug was administered topically at $0.05 \%$ and $0.18 \%$ concentrations. The drug was applied twice a day for 3 consecutive months; then it was suspended for 1 month, and the biopsy sample was repeated for the histological follow-up. The higher concentration of the drug, according to the same protocol, was administered to patients who did not benefit from the lower concentration. The results showed a significant reduction in lesions (85\%), with no documented topical or systemic adverse reactions at $0.18 \%$ concentration. A significant reduction of the aggressiveness of the disease and the disappearance of dysplastic phenomena were observed histologically. The proposed therapeutic protocol was effective toward highly active oral leukoplakia with dysplastic phenomena, and therefore at higher risk of malignant progression. 02006 Prous Science. All rights reserved.

Key words: Isotretinoin - Oral leukoplakia therapy

\section{INTRODUCTION}

The biological effectiveness of vitamin $\mathrm{A}$ has been known since the beginning of the 20th century even if, at first, the studies were focused on its deficiency with consequent night blindness. Vitamin A plays a complex biological role through the action of three active metabolic agents: retinoic acid, retinal and retinaldehyde. While aldehyde is mainly involved in the processes connected with vision and reproduction, retinol, and particularly retinoic acid, intervene in organogenesis and in oral mucosa tissue tropism. Retinol is present in numerous phases of epithelial tissue growth, differentiation and maintenance $(1,2)$.

A thorough accurate literature review has shown that vitamin A and its derivatives have very important biological functions (3-5). It regulates epithelial differentiation, influences the immune system and some phlogosis mechanisms, and has a direct action on intercellular cohesion and interaction. Vitamin A deficiency can reveal itself with the formation of keratinized epithelium layers in correspondence with the oral mucosa while, in contrast, its differentiation can be inhibited by an excess (6). In vitro, vitamin $\mathrm{A}$ inhibits the malignant transformation caused by chemical carcinogenic agents, ionizing radiations and viruses by interfering with the first phase of epithelial transformation and this preventive effect has proven useful even for oral mucosa cells (7).

It has been observed that the promoting factors of tumoral forms increase the activity of one enzyme: ornithine decarboxylase. The latter limits polyamine synthesis involved in cell proliferation and differentiation. The retinol metabolic agents, instead, reduce ornithine decarboxylase activity. The induction of the latter probably occurs during the G1 phase of the cellular cycle and therefore the effects of retinol are most likely represented by the ability to block the cellular cycle in the G1 phase, due to ornithine decarboxylase inhibition. Supporting this thesis is the observation that the treatment of melanomatous cellular lines with retinoic acid increases the ratio in the G1 phase of their own cycle. Retinol shows a greater therapeutic effect before tumoral development (8-11).

Vitamin A also influences tumoral and cellular immunity: low doses of retinoic acid stimulate a cellmediated response, whereas high doses inhibit it.

Retinoic acid and its derivatives induce the activation of macrophages, making them highly cytostatic, with the consequent release of arginase, considered an important barrier against tumoral cells. The role played by retinol on the macrophages may be the result of 
prostaglandin inhibition, since they inhibit tumoricidal macrophage activity $(1,3,6)$.

Glycosaminoglycan synthesis is also influenced by retinoids. Since these glycoconjugated agents are involved in cellular recognition and bonding, this may be a way through which retinoids influence differentiation, but also the correct balance of oral epithelium (10).

Following the studies, which have shown the important biological effects of vitamin $\mathrm{A}$ and its derivatives, our study aimed to evaluate the therapeutic effects of isotretinoin at different concentrations $(0.05 \%$ and $0.18 \%$ ) in the management of oral leukoplakia (OL).

Several studies have been carried out in the past, which aimed to evaluate the role of vitamin A derivatives on OL (1-11), but our study is characterized by histological monitoring before and after the treatment, by the 10 -year follow-up of 20 cases, by the early evaluation of relapse, and by the posology and method of administration of the therapy.

\section{MATERIALS AND METHODS}

Forty patients (M/F: 18/12; mean age: $56.69 \pm 5.4$; range: 48-65), who, from 1994 to 2004, came to our department for diagnostic investigations with a diagnosis of suspected OL, were examined. Patients were always followed-up by the same physician. After thorough anamnesis and clinical examination, and having confirmed the diagnosis of suspected OL, a histological sample was taken for the final confirmation of the diagnosis. The biopsy was carried out under local anesthesia with the punch technique in all the patients examined.

After the confirmation of the instrumental diagnosis of OL, patients were included in the study if they gave their written consent after being properly informed about the therapeutic aspects of the experimental protocol. Of the 40 patients involved, 29 were smokers and none of five alcohol drinkers were spirit drinkers. All the patients were informed about the importance of avoiding risk factors, such as smoking and alcohol, in order to reduce the possibility of lesion progression and to make the treatment effective.

The patients were divided into two groups: group A, comprising 20 patients, was administered a galenic preparation based on $0.18 \%$ isotretinoin; group B, with 20 patients, was also administered a therapy based on isotretinoin, but at a lower concentration, i.e., $0.05 \%$. The patients were randomly assigned to one or the other group, since they were selected in order of presentation to our department. The drug was administered topically twice a day for 3 consecutive months; then it was suspended for 1 month and the biopsy sample was repeated for the histological follow-up. The medication used was not known to the patient or the clinician. All the patients were advised to apply the drug to a gauze and to apply this to the lesion site. The use of gauze permits the application for sufficient time, reducing salivary inter- ference. The patients were advised to keep the gauze in place for at least 10 consecutive minutes. The application was carried out twice a day, in the morning after breakfast and in the evening after dinner and after thorough oral care. After 3 consecutive months of therapy and after clinical and histological observations, the patients to whom the drug at lower concentration had been administered and who did not show a clinical and histological improvement of the disease, were treated with the drug at higher concentration according to the same therapeutic protocol and were then evaluated again both clinically and histologically.

Before starting therapy, all lesions were given a clinical score by classifying the severity of OL. The score ranged from 0 to 3 as follows: $3=$ white plaque $>2 \mathrm{~cm}$; $2=$ white plaque $>1 \mathrm{~cm}$ and $<2 \mathrm{~cm} ; 1=$ white plaque $<1 \mathrm{~cm} ; 0=$ normal mucous. The visual analog scale was used for the pain score. Pain scores were from 0 (no pain) to 10 (extreme pain). The Wilcoxon rank sum test was used for the analysis of the sign scores.

The Galenical preparation was made in our laboratories in a dark room and stored in containers protected from light by tinfoil. The drug was prepared at two different concentrations: $0.05 \%$ concentration, used in many studies reported in the literature and a higher $0.18 \%$ concentration, chosen through experience. Isotretinoin, taken from capsules (Roaccutan, Roche, Milan, Italy) was included in $50 \mathrm{~g}$ of carboxy-methylcellulosebased gel (Oralbalance gel, Biopharm, Peschiera, Italy). After taking the isotretinoin from the capsules by means of $5 \mathrm{ml}$ syringes, the active principle was included in the gel; subsequently, the preparation was homogenized. Then, the patients were advised to store the Galenic preparation in the refrigerator.

During the first 3 months of treatment, the patients were followed-up every 15 days to evaluate the clinical progress of the lesion and, above all, to check the methods of application of the drug, which was prepared again before it was finished, as well as to evaluate any appearance of undesired effects. Subsequently, the treatment was interrupted for 1 month and each patient was evaluated both from the clinical and the histological point of view, to monitor any lesion modifications. The patients continued to be followed-up to evaluate the preservation of the results obtained and any lesion relapse following therapy interruption. A group of 20 patients had a 10-year follow-up.

\section{RESULTS}

The 40 patients with OL had different clinical and histological features. Thirteen had a score of 3,20 had a score of 2 , and 7 had a score of 1 . Of the latter, five showed dysplasia in progress or very high disease activity. In all the patients, this was located in correspondence with the gingival mucosa and also with the dorsal tongue in 22 cases. 

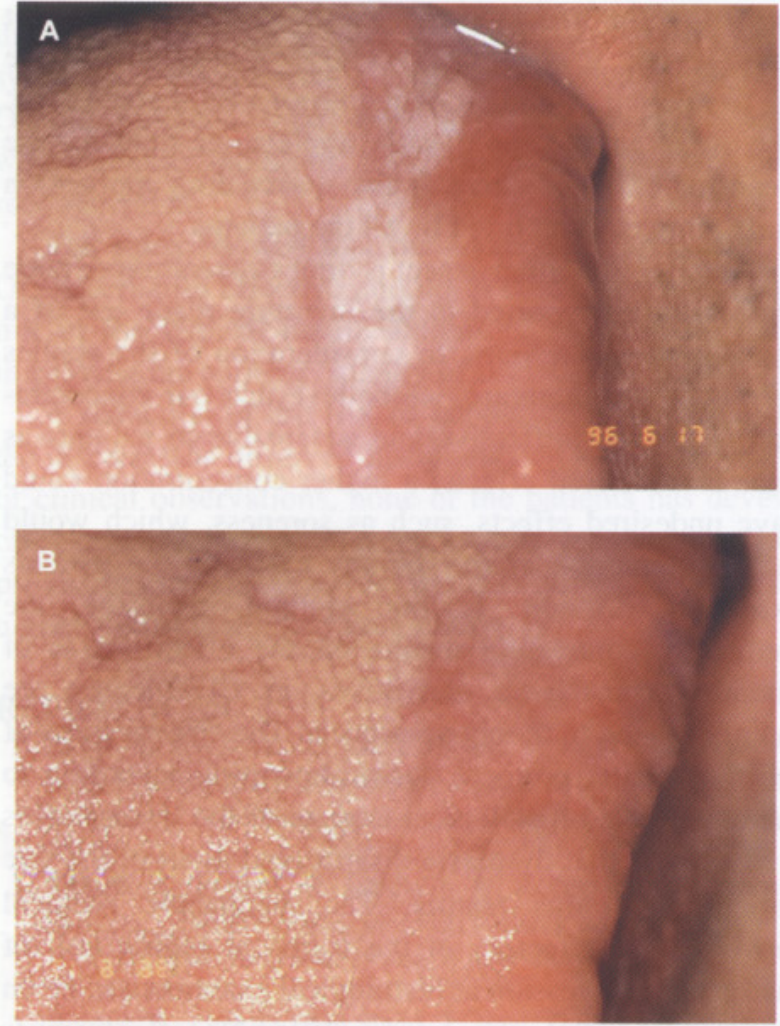

FIG. 1. Clinical features before (A) and after (B) therapy (isotretinoin $0.18 \%$ ) (magnification: $\times 25$ staining method: hematoxylin-eosin).

There were no statistically significant differences in clinical severity between the two groups $(p>0.01)$.

The symptomatology was absent in all the patients (pain scores $=0$ ). The patients with lingual localization reported taste alteration.

Following the application of the Galenic preparation, the modifications of the clinical-histological pictures were different both according to drug concentration and clinical characteristics of the disease.

In all the patients observed, topical application of the drug produced, during the first $30 \mathrm{~min}$, an increase of soreness and pain and greater sensitivity to hot foods. However, the symptomatology was transitory and considered acceptable by the patients. No other undesired effects of the drug were reported by the patients, independently of its concentration.

In the group of patients subjected to therapy with the drug at higher concentration, seven had a score of 3,12 had a score of 2 and one had a score of 1 .

After 3 consecutive months of treatment, in 14 patients the oral hyperkeratosis disappeared (Fig. 1). At a histological level dysplasia disappeared in the three cases belonging to this group (Fig. 2).

In the second group of patients who received the drug at the lower concentration, six had a score of 3 , nine had a score of 2 and five had a score of 1 . After 3 consecutive months of treatment, in nine patients the oral hyperkeratosis disappeared. At a histological level
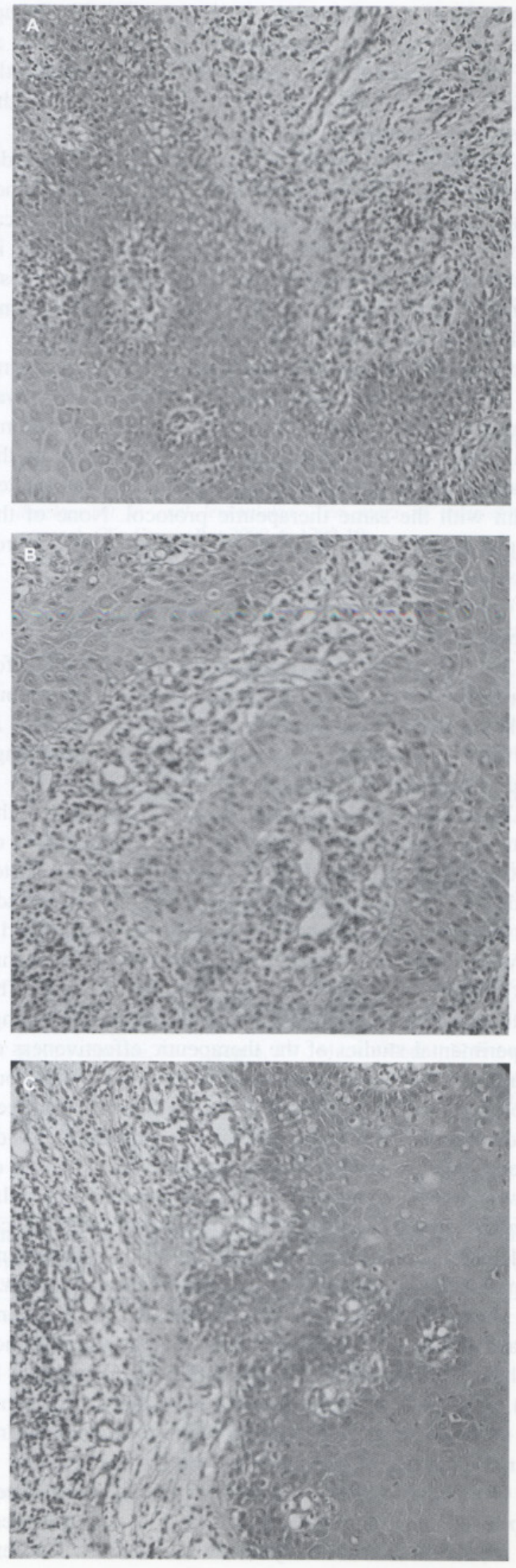

FIG. 2. Histological appearance before (A, B) and after (C) therapy (isotretinoin $0.18 \%$ ) (magnification: $\times 25$ staining method: hematoxylin-eosin). 
dysplasia remained in the two patients in this group. The change noted in the patients receiving the drug at the higher concentration was statistically significantly greater than that in the patients receiving the drug at the lower concentration $(p<0.01)$.

The 11 patients with no clinical-histological modifications were administered the drug at the higher concentration. In this case, clinical and histological improvement was observed in eight patients. Even in this case, the two patients with dysplasia at the posttherapy histological follow-up showed an improvement, with the disappearance of dysplastic phenomena.

Of the 40 patients observed and followed-up during the 10 years of the study, the first original group was composed of 20 patients (three with dysplastic phenomena). They are still under observation; a relapse of the lesion occurred in only four patients and was treated again with the same therapeutic protocol. None of the 40 patients involved in the study showed malignant progression of the lesion.

\section{DISCUSSION}

The treatment of OL remains a real challenge for clinicians who deal with this patient population and thus with the diagnosis of this disease. Before a patient is started on therapy, a biopsy must be done and the diagnosis established (12-16).

The most effective treatment modality to control the signs and symptoms of the disease is short courses of systemic steroids and topical high-potency corticosteroids $(11,13,14,17,18)$. Other forms of therapy include the use of retinoids, both systemic and topical (11). However, there is no one single standard protocol that has been proven effective with systemic retinoids. The results so far are controversial and not very encouraging. Experimental studies of the therapeutic effectiveness of vitamin $\mathrm{A}$ and its derivatives have shown a continuous evolution. Indeed, over the years, an attempt has been made to reduce the limits of their use and thus their side effects, mainly aiming at three objectives: synthesis of new vitamin derivatives, which induce lower accumulation on adipose tissue, and lower organism permanence, and therefore also reduction of undesired effects, and minimum administered dose-method of administration.

Vitamins, such as A, $\beta$-carotene, C, E, B12 and folate, are the micronutrients with the strongest evidence of having a link to cancer prevention and control $(7,8$, 19). Deficiency of these vitamins at the dietary, systemic or mucosal level will interact with tobacco use and increase the risk of oral precancerous lesions $(20,21)$.

Over the years, different opinions have been expressed regarding the effectiveness of OL treatment by means of vitamin A synthetic derivatives. Two extremely important, innovative aspects introduced in our study are represented both by the method of application of the drug and the concentration of the preparation.
Regarding the first aspect, since the oral cavity is a wet environment, it is not easy to apply a drug and, above all, to allow it to remain on the lesions for a given time. The use of gauze simplifies the application, reducing the cleansing effect of saliva. It also makes drug application fairly simple, as reported by all the patients.

Another important aspect is drug concentration; none of the studies reported in the literature made use of a concentration like ours. In consideration of the studies present in the literature, we believe that our concentration is the most effective and acceptable to patients as regards undesired effects; a higher concentration could have undesired effects, such as soreness, which would make completion of the therapy cycle by the patients extremely difficult. In our opinion, therefore, the frequently contrasting results on the effectiveness of vitamin A synthetic derivatives in OL therapy are mainly related to these two aspects. The effectiveness of the therapeutic protocol varies, however, according to the clinical and histological aspects of the lesions. This study shows that dysplasia disappeared in all of the five patients with this feature. The therapeutic protocol proved more effective in the forms at higher risk of malignant progression at histological examination. From the clinical point of view, considerable effectiveness was observed in the resolution of hyperkeratosis.

Certainly, the most important aspect of the study is that none of the patients observed progressed to oral carcinoma. This is an important result, showing the effectiveness of the therapy, mainly in preventing disease progression.

Another aspect worth mentioning is that of disease relapse. Over the years several patients gradually showed a worsening of the disease both clinically and histologically. These "relapses,"' however, were always managed with the same therapeutic approach. Therefore, according to us, a therapeutic treatment is effective or ineffective; the relapse must be connected with the progress of the disease itself. The element showing treatment effectiveness was that malignant progression of precancer occurred in none of the patients; on the contrary, histologically, we observed that our protocol reduces the probability of neoplastic progression, reducing both dysplasia and disease activity (22-27).

Another aspect, which characterizes our therapeutic approach, is the administration method, which is topical. This is important because the undesired effects of the therapy are reduced and made exclusively local and limited in time. Moreover, topical administration virtually eliminates the risk of teratogenesis, common in every therapy with vitamin A derivatives. In fact, it is known that the systemic administration must be avoided in fertile women and followed by contraceptive therapy for at least 2 years to avoid this risk, since retinoids are deposited at the level of adipose tissue and slowly released. Moreover, topical administration makes the 
therapeutic protocol applicable in all patients in whom systemic corticosteroid treatment is contraindicated. Of our patients, eight were hypertensive and seven were diabetic; therefore, corticosteroid therapy was not recommended.

The study showed that our therapeutic approach to $\mathrm{OL}$ is certainly effective and up to date. It permits control of the clinical manifestations of the disease, thus improving the patients' quality of life.

A reduction of the risk of neoplastic progression of the disease was observed histologically; in the 10 years of clinical observations, none of the patients has developed oral carcinoma. The therapeutic protocol applied proved particularly effective in the histological forms at higher risk of malignant progression.

\section{REFERENCES}

1. Beenken, S.W., Sellers, M.T., Huang, P. et al. Transforming growth factor-alpha (TGF-alpha) expression in dysplastic oral leukoplakia: Modulation by 13-cis retinoic acid. Head Neck 1999, 21: 566-73.

2. Piattelli, A., Fioroni, M., Santinelli, A., Rubini, C. blc-2 expression and apoptotic bodies in 13-cis retinoic acid (isotretinoin)topically treated oral leukoplakia: A pilot study. Oral Oncol 1999, 35: 314-20.

3. Boisnic, S., Licu, D., Ben Slama, L. et al. Topical retinaldehyde treatment in oral lichen plainus and leukoplakia. Int J Tissue React 2002, 24: 123-30.

4. Brachet, M.C., Boisnic, S., Pascal, F. et al. Topical tretinoin in the treatment of lichen planus and leukoplakia of the mouth mucosa. A biochemical evaluation of the keratinisation. Ann Dermatol Venereol 1994, 121: 459-63.

5. Garewal, H.S., Meyskens, F.L., Killen, D. et al. Response of oral leucoplakia to beta-carotene. J Clin Oncol 1990, 8: 1715-8.

6. Garewal, H. Chemoprevention of oral cancer: Beta-carotene and vitamin E in leukoplakia. Eur J Cancer Prev 1994, 3: 101-7.

7. Huber, M.H., Hong, W.K. Biology and chemoprevention of head and neck cancer. Curr Probl Cancer 1994, 18: 81-140.

8. Reichart, P.A. Identification of risk groups for oral precancer and cancer and preventive measures. Clin Oral Investig 2001, 5: 207-3.

9. Tete, S., Pappalardo, S., Rubini, C., Salini, L., Falco, A., Perfetti, E.G. The role of apoptosis and blc-2 protein in topical treatment of oral leukoplakia with isotretinoin. Minerva Stomatol 1999, 48: 411-8.

10. Tradati, N., Grigolat, R., Calabrese, R. et al. Oral leukoplakia: To treat or not? Oral Oncol 1997, 33: 317-21.

11. Lodi, G., Sardella, A., Bez, C. et al. Interventions for treating oral leukoplakia (Cochrane Review). Cochrane Database Syst Rev 2001, 4: CD001829.
12. Ben Slama, L. Precancerous lesions of the buccal mucosa. Rev Stomatol Chir Maxillofac 2001, 102: 77-108.

13. Fettig, A., Pogrel, M.A., Silverman, S. Jr. et al. Proliferative verrucous leucoplakia of the gengiva. Oral Surg Oral Med Oral Pathol Oral Radiol Endod 2000, 90: 723-30.

14. Kahn, M.A., Docketer, M.E., Hermann-Petrin, J.M. Proliferative verrucous leukoplakia four cases with flow cytometric analysis. Oral Surg Oral Med Oral Pathol Oral Radiol Endod 1994, 78: 469-75.

15. Scully, C., Cawson, R. Potentially malignant oral lesions. J Epidermid Biostat 1996, 1: 3-12.

16. Shepmore, K.P., van der Meij, E.H., Smeele, L.E.van der Waal, I. Prevalence study of oral white lesions with special reference to a new definition of oral leukoplakia. Eur J Cancer B Oral Oncol 1996, 32: 416-9.

17. van der Waal, I., Schepman, K., van der Meij, E.H. et al. Oral leukoplakia: A clinicopathological review. Oral Oncol 1997, 33: 291-301.

18. Vuckovic, N., Boko-Bratic, M., Vuckovic, D. Histological characteristics of oral leukoplakia. Med Pregl 2004, 57: 140-3.

19. Lee, J.J., Hong, W.K., Hittelman, W.N. et al. Predicting cancer development in oral leukoplakia: Ten years of translational research. Clin Cancer Res 2000, 6: 1702-10.

20. Axell, T., Pindborg, J.J., Smith, C.J. et al. Oral white lesions with special reference to precancerous and tobacco-related lesions: Conclusions of an international symposium held in Uppsala, Sweden, May 18-21, 1994. J Oral Pathol Med 1996, 25: 49-54.

21. Batsakis, J.G., Suarez, P., el-Naggar, A.K. Proliferative verrucous leukoplakia and its related lesions. Oral Oncol 1999, 35: 354-9.

22. Shin, M.N., Chen, T.H. Intervention efficacy and malignant transformation to oral cancer among patients with leukoplakia (review). Oncol Rep 2003, 10: 1683-92.

23. Kleier, C., Werkmeister, R., Joos, U. Zinc and vitamin A deficiency in diseases of the mouth mucosa. Mund Kiefer Gesichtschir 1998, 2: 320-5.

24. Bagan, J.V., Jimem, Y., Sanchis, J.M. et al. Proliferative verrucous leucoplakia: High incidence of gingival squamous cell carcinoma. J Oral Pathol Med 2003, 32: 379-82.

25. Bouquot, J.E. Oral cancer with leucoplakia. Oral Dis 1999 , 5: $183-4$.

26. Boyle, P., Chiesa, F., Scully, C. Chemoprevention and oral cancer: (More) trials and (more) tribulations. Eur J Cancer B Oral Oncol 1995, 31: 1-2.

27. Haya-Fernandez, M.C., Bogon, J.V., Mucillo-Cortes, J., ProvedaRada, R., Calabuig, C. The prevalence of oral leukoplakia in 138 patients with oral squamous cell carcinoma. Oral Dis 2004, 10: $346-8$.

Address for correspondence: Dr. Giuseppe Alessandro Scardina, Department of oral science, University of Palermo, via del Vespro, 129-90127, Palermo, Italy. E-mail: scardina@osdonto.unipa.it 